\title{
X-linked intellectual disability, Snyder type
}

INSERM

\section{Source}

INSERM. (1999). Orphanet: an online rare disease and orphan drug data base. $\underline{X \text {-linked }}$ intellectual disability, Snyder type. ORPHA:3063

X-linked intellectual disability, Snyder type is a rare X-linked intellectual disability syndrome characterized by hypotonia, asthenic build with diminished muscle mass, severe generalized psychomotor delay, unsteady gait and moderate to severe intellectual disability, as well as a long, thin, asymmetrical face with prominent lower lip, long fingers and toes and nasal, dysarthric or absent speech. Bone abnormalities (e.g., osteoporosis, kyphoscoliosis, fractures, joint contractures) are also characteristic. Myoclonic, or myoclonic-like, seizures and renal abnormalities have been associated in some patients. 